\title{
CEREBELLAR ATROPHY IN LEUKEMIA SURVIVORS
}

MRI changes in the cerebellum and cognitive function of 13 survivors of childhood acute lymphoblastic leukemia (ALL) treated with cranial radiation of $24 \mathrm{~Gy}$ and intrathecal methotrexate were studied at the University of New Mexico, Albuquerque, Manitoba Cancer Foundation, Winnipeg, and University of Alberta, Edmonton. Age at diagnosis was a mean of 3 yrs 3 mos (range, 2 yrs - 4 yrs 10 mos), and age at testing was 11 yrs 8 mos $(9-14$ yrs). Hypoplasia of the cerebellar vermis and cognitive deficits involving visualspatial-motor coordination and memory were observed in patients compared to controls. (Ciesielski KT et al. Hypoplasia of the cerebellar vermis and cognitive deficits in survivors of childhood leukemia. Arch Neurol Oct 1994;51:985-993). (Reprints: Dr Ciesielski, Department of Psychology, University of New Mexico, Logan Hall, Albuquerque, NM 87131).

COMMENT. The neurotoxic effects of prophylactic cranial irradiation in children with acute lymphoblastic leukemia are well documented. As in this study, deficits in visual-motor skills and right brain involvement have been more pronounced than language and verbal deficits evidence of left brain sparing. A causal relationship between the structural cerebellar abnormalities and the neuropsychological dysfunction is unproven. Children treated before 5 years of age are more susceptible to post-irradiation cognitive deficits than those diagnosed at or later than 5 years. Children in families of higher educational status who receive more attention and academic stimulation have a lower incidence of cognitive deficit than those with sensorymotor learning deprivation.

The dose of cranial irradiation, 2400 rads, used in this study is highly toxic in young patients. In the 1980 s, most leukemia protocols limited the prophylactic dose to 1800 rads for use only in high risk patients. Reliance on intrathecal methotrexate chemotherapy for the majority of ALL patients is not known to result in significant intellectual or coordination deficits. (Personal communication: Dr David O. Walterhouse, Oncology Service, Children's Memorial Hospital, Chicago).

\section{MOVEMENT DISORDERS}

\section{HEREDITARY ESSENTIAL TREMOR}

Twenty index patients with hereditary essential tremor, and 93 first degree and 38 more distant relatives were studied at the Institute of Neurology, Queen Square, London, and Oldchurch Hospital, Romford, UK. Tremor presented in the arms. It was symmetrical in $75 \%$ and first noted in the dominant hand in $25 \%$. In index patients, tremor spread to affect the legs, head, voice, tongue, face, and rarely the jaw. Affected relatives had tremor of the upper limbs, and spread occurred in a minority. The median age of onset for index patients was 15 yrs (range 5 - 52). The age at onset for both index and secondary cases was bimodal, peaking in the second and fifth and sixth decades. Of 14 relatives under 15 years old, 4 were described as tremulous and 2 had definite tremor, beginning as early as 2 years of age. Disability occurred in the majority but none before 15 years of age. Hunger, emotion, fatigue, and heat exacerbated tremor and disability, whereas alcohol was of benefit in 50\%. Classical 
migraine was associated in $25 \%$ cases. Dystonia, included in some previous studies, was not encountered. Inheritance was autosomal dominant and penetrance was complete by age 65 . (Bain PG, Marsden CD et al. A study of hereditary essential tremor. Brain 1994;117:805-824). (Respond: Dr PG Bain, MRC Human Movement and Balance Unit, Institute of Neurology, Queen Square, London WC1N 3BG, UK).

COMMENT. The diagnosis should be considered in older children described as tremulous or having a mild symmetrical postural tremor of the upper limbs. Genetic counselling is pertinent in a parent with tremor and a currently unaffected child. The authors' data show that at birth, the risk of ever developing essential tremor is $46 \%$, and the risk of being affected by 20 years is $30 \%$. In an unaffected child of 15 years of age, the risk of tremor by 20 years is $8 \%$, and by 40 years, $20 \%$.

\section{MIGRAINE AND RELATED DISORDERS}

\section{ASPARTAME-INDUCED HEADACHE}

A double-blind crossover study in 32 subjects with self-identified aspartame-induced headache is reported from the University of Washington School of Medicine, Seattle, WA. Volunteers were randomized to receive aspartame $(30 \mathrm{mg} / \mathrm{kg} / \mathrm{d})$ and placebo in a 2-treatment, 4-period crossover design. Each period was 7 days. Subjects reported significantly more headaches during aspartame treatment (on $33 \%$ of the days) compared with placebo (24\%). Headache triggered by aspartame was particularly frequent [p $<0.001]$ in subjects who were "very sure" that aspartame had caused them headaches previously. One-fourth of the subjects withdrew from the study, complaining of too frequent or severe headaches or sleep disturbance. A number of individuals had declined inclusion in the study because of the severity of their reaction to aspartame. (Van Den Eeden SK et al. Aspartame ingestion and headaches: A randomized crossover trial. Neurology Oct 1994;44:1787-1793). (Dr SK Van Den Eeden, Division of Research, Kaiser Permanente Medical Care Program, 3505 Broadway, Oakland, CA 94611).

COMMENT. The authors conclude that aspartame causes headaches in a subset of adults with self-identified aspartame-induced headaches.

An underestimation of the adverse effect of aspartame in some studies may reflect differences in subject susceptibility, exclusion of specific responders, and concomitant ingestion of other food or drink. Children with migraine may be more responsive to dietary triggers than adults. (Progress in Pediatric Neurology I \& II, Chicago, PNB Publ, 1991, 1994).

\section{BENIGN NOCTURNAL ALTERNATING HEMIPLEGIA}

Two brothers who developed recurrent attacks of alternating hemiplegia arising out of sleep, distinguishable from classical alternating hemiplegia of childhood (AHC), are reported from Montreal Neurological and Children's Hospitals, and Children's Hospital of Western Ontario, London, ON, Canada. Both infants awoke screaming $11 / 2$ hrs after falling asleep and were paralyzed on one side. After returning to sleep and awakening in the morning, they had recovered. Similar episodes recurred with increasing frequency. Features such as hypotonia, dystonia, and eye movements, characteristic of AHC, were absent. Development remained normal. Both 